\title{
Economic valuation of the potential health benefits from foods enriched with plant sterols in Canada
}

\author{
Collin L. Gyles', Jared G. Carlberg', Jennifer Gustafson², \\ David A. Davlut ${ }^{2}$ and Peter J.H. Jones ${ }^{2}$
}

\author{
'Department of Agribusiness \& Agricultural Economics, University of Manitoba, Winnipeg, Canada; ${ }^{2}$ Richardson \\ Centre for Functional Foods and Nutraceuticals, University of Manitoba, Winnipeg, Canada
}

\section{Abstract}

Background: Increased consumption of foods containing plant sterols has the potential to reduce the incidence of coronary heart disease (CHD) and thus reduce costs associated with treating that disease in a significant way. This paper reports the results of an investigation of the potential monetary benefits of allowing foods enriched with plant sterols to be marketed in Canada.

Objective: The objective of this research was to estimate the annual savings that would accrue to Canada's single-payer publicly funded health care system if plant sterols were approved for use. If foods containing plant sterols are consumed at a sufficient rate, a reduction in CHD should follow. Given the significant costs associated with CHD, approval of plant sterols in Canada has important public policy implications.

Design: This research employs a variation of traditional cost-of-illness analysis entailing four steps: (1) estimation of a 'success rate' (proportion of persons who would consume plant sterols at the necessary rate); (2) presumption of blood cholesterol reduction due to plant sterol consumption; (3) assumption of reduction in $\mathrm{CHD}$ that follows from blood cholesterol reduction; and (4) calculation of cost savings associated with reduced incidence of CHD.

Results: Calculations were carried out for four scenarios: ideal, optimistic, pessimistic, and very pessimistic. It was estimated that between $\$ 38$ million (very pessimistic scenario) and $\$ 2.45$ billion (ideal scenario) could be saved annually by Canada's health care system with plant sterol-enriched food products being made available for sale.

Conclusion: Significant expenditure reductions within Canada's publicly funded health care system could be realized with plant sterols approved for sale. Reduced CHD resulting from lower blood cholesterol levels would lessen the financial burden of disease in Canada.

Keywords: coronary heart disease; cost-of-illness analysis; health care costs; success rate

Received: 2 March 20I0; Revised: II May 20I0; Accepted: 3 September 2010; Published: 7 October 2010

$\mathrm{T}$ he potential health benefits gained from consuming plant sterol-enriched foods have been studied as early as the 1950s (1), with the preponderance of literature indicating that plant sterol and stanolenriched food can reduce blood cholesterol in humans (2-5). In 2006, new harmonized regulations were adopted by the European Union (EU) regarding use of health claims and nutrition claims for food products; the new legislation requires clear scientific substantiation of all such claims. Recently, the European Food Safety Authority approved the claim that 'Plant sterols have been shown to lower/reduce blood cholesterol. Blood cholesterol lowering may reduce the risk of (coronary) heart disease' (6).

High blood cholesterol is a major risk factor for heart disease and stroke; individuals with high blood cholesterol have a greater likelihood of developing coronary heart disease (CHD). Thus, if consumption of foods containing plant sterols can reduce the number of individuals with high cholesterol, it follows that consumption of plant sterols should lead to a corresponding decrease in the incidence of CHD. Statistics Canada in 1998 estimated outlays for physician services, hospital costs, lost wages, 
decreased productivity, and other losses attributed to CHD in Canada to be approximately $\$ 18$ billion (7). Accordingly, it is expected that a lowering of the incidence of heart disease will result in a reduction of some of the costs associated with treating CHD.

In Canada, as in other jurisdictions with publicly funded health care systems, the rising costs of treating diseases and the economic production lost due to premature death and disability are a major concern. The potential economic impact of incorporating plant sterols into Canadians' diets can be calculated by determining the proportion of the estimated $\$ 18$ billion cost of CHD avoidable by allowing public consumption of plant sterols in Canada. Previous research has shown that consumption of plant sterol-enriched margarine could result in significant benefits from the perspective of a European health insurer (8); however, no comparable research has been done in a Canadian context to date. A previous attempt to estimate the potential economic impact in Canada from the introduction of trans-fatfree canola oil showed a significant reduction in the costs associated with CHD (9). A similar approach could be taken with respect to plant sterol-enriched food.

The purpose of this research is therefore to determine the potential economic savings associated with allowing plant sterol consumption in Canada. The process to establish the potential value of these economic savings from the consumption of plant sterol-containing foods involves four steps: (1) assessment of a 'success' rate; (2) presumption of a possible blood cholesterol reduction due to consumption of foods containing plant sterols; (3) assumption of the reduction in the incidence of CHD due to the realized decrease in blood cholesterol levels; and (4) calculation of the reduction in costs associated with decreased prevalence of CHD. Results provide an early approximation of the potential health care savings associated with use of plant sterol-enriched foods; it is expected that future research using more elaborate techniques will yield more refined estimates.

\section{Methods}

When attempting to measure potential health benefits, a number of methods are available including cost-benefit analysis, cost-effectiveness analysis, and cost-of-illness analysis. The selection of an appropriate method should be consistent with the available data and the phenomenon being studied. Cost-benefit analysis and cost-effectiveness analysis are both used for evaluation of two or more alternative treatments; these techniques require the costs associated with both the treatment and the alternatives to be well-known. The purpose of this research is to determine the potential benefits from allowing plant sterol-enriched foods to be sold into a market where they are currently not available, not to compare plant sterolenriched foods to alternative treatments. Additionally, it is not possible to estimate the cost premium that would be attached to foods enriched with plant sterols in Canada as this market would need to evolve in order to establish a long-term price. As such, neither cost-effectiveness nor cost-benefit analysis is an appropriate tool for this analysis.

A cost-of-illness analysis measures the direct and indirect costs of a specific disease - in this case CHD. A variation of a cost-of-illness analysis is to calculate the dollar savings associated with preventing or avoiding an illness. This variation has been used to calculate the economic benefit of drug abuse interventions (10), preventable drug-related medical complications (11), and trans-fat-free canola oil (9). A similar approach to the technique employed here was used previously by Malla, Hobbs, and Perger to estimate health care savings from trans-fat-free canola oil in Canada (12).

The economic benefit of consumption of plant sterolcontaining foods is evaluated using an economic simulation involving four steps, described below.

\section{Step I: assessment of a 'success rate'}

In order to realize health benefits from plant sterols, an individual must consume between 1 and $3 \mathrm{~g} / \mathrm{day}$. The proportion of the Canadian population that will consume a sufficient quantity of plant sterol-enriched foods to reduce their cholesterol level is defined as the success rate. Plant sterols can feasibly be added to a number of food products such as orange juice, cheese, milk, yogurt, salad dressing, cereal products, cooking oils, and margarine. According to Statistics Canada, the average Canadian consumption of these food categories is around nine servings per day (13). It is thus reasonable to conclude that there is sufficient scope within the Canadian diet to accommodate an adequate dose of plant sterols without having to drastically alter food consumption patterns. It should be noted that all of the foods listed above may not be equally effective as delivery vehicles for plant sterols; it may also be the case that claims will be allowed for different foods containing plant sterols along different timelines.

Since the market for plant sterol-enriched foods has not until very recently existed in Canada, estimating its size and characteristics is difficult. The overall Canadian functional food market, of which phytosterol fortified foods will become a subset, is still evolving and changing rapidly and thus available data are relatively sparse. Given the lack of available information, the best method for predicting how Canadians would respond to plant sterol-enriched food products is to examine the markets for similar functional foods in Canada and plant sterolenriched foods in other countries.

An activities, interests, and opinions (AIO) framework and cluster analysis was used to determine the potential markets for functional foods in Canada (14). Survey 
information from across Canada enabled a cluster analysis to determine attitudes, knowledge, and motivations combining to identify consumers likely to buy functional foods. It was discovered that a cluster representing approximately $47 \%$ of the adult Canadian population possesses the characteristics that would make them likely to purchase functional food products. Canadian survey data available to determine the acceptance of specific functional foods are limited when compared to larger markets; however, the acceptance of conjugated linoleic acid (CLA) fortified foods has been examined in Western Canada (15). It was found that the high percentage of survey respondents likely to purchase CLA-enriched dairy products, if available where they normally shop, ranged from $4 \%$ to $13 \%$. Since many of these dairy products are similar to potential phytosterolfortified foods, it seems reasonable to extend these results to such products.

In Finland, a country which has allowed phytosterol/ stanol-fortified foods in the marketplace for quite some time, a survey found $17 \%$ of adults reported daily use of plant sterol-enriched margarine, with another 20\% reporting monthly use (16). An additional 7\% indicated having occasionally used the product. For the purpose of this economic evaluation, the success rates shown in Table 1 were used in the various sensitivity analysis scenarios as an estimate of the success ratio.

\section{Step 2: presumption of a possible blood cholesterol reduction due to consumption of foods containing plant sterols}

The primary health benefit of consuming foods fortified with phytosterols is a reduction in serum cholesterol levels. Approximating this reduction requires extrapolation of results of meta-analyses of available human phytosterol-feeding studies. Since plant sterols are relatively well-established as functional food additives, several thorough meta-analyses exist (2-5). One of these was limited in scope to plant sterol-enriched spreads and found a reduction in LDL-cholesterol of 8.4\% (95\% CI 6.6-10.0\%) (3). Another included 23 plant sterol and stanol trials and found a serum cholesterol reduction

Table 1. Estimated plant sterol success rates

\begin{tabular}{|c|c|c|}
\hline Assumption & $\begin{array}{c}\text { Plant sterol } \\
\text { consumption rate (\%) }\end{array}$ & Source \\
\hline $\begin{array}{l}\text { Full potential all consumers } \\
\text { who are predisposed to } \\
\text { purchase functional food }\end{array}$ & 47 & Herath et al. (14) \\
\hline $\begin{array}{l}\text { Consumption level similar } \\
\text { to Europe }\end{array}$ & 17 & Niva (16) \\
\hline $\begin{array}{l}\text { Consumption level similar } \\
\text { to CLA survey intentions }\end{array}$ & 10 & Peng et al. (I5) \\
\hline
\end{tabular}

ranging between 4.6 and $24.3 \%$ with a mean LDLcholesterol reduction of $11.0 \%$ (4). Yet another used 21 published plant sterol studies found that average dose of $2.3 \mathrm{~g}$ of plant sterols per day yielded an average cholesterol reduction of $9.7 \%$ (95\% CI 8.5-10.8\%) (2). The most recent meta-analysis examined the results of 59 studies and found that plant sterol containing products reduced LDL-cholesterol by $0.31 \mathrm{mmol} / \mathrm{L}$, a significant reduction (5).

The results from the meta-analysis based on 21 published plant sterol studies (2) were adopted for the majority of the scenarios in the sensitivity analysis. This work included the largest number of trials limited exclusively to plant sterol-enriched foods and did not include plant stanol-enriched foods. As a result, this dataset was judged as best representing the relationship between plant sterol consumption and serum LDLcholesterol level reduction in humans.

Step 3: assumption of the reduction in the incidence of $\mathrm{CHD}$ due to decrease in blood cholesterol levels

Since no long-term studies have directly tracked the relationship between phytosterol consumption and heart disease in humans, it was necessary to make assumptions regarding the rate at which lower cholesterol levels due to plant sterol consumption will reduce the incidence of CHD. A meta-analysis of cholesterol-reducing treatment studies found a 13-14\% reduction in CHD mortality for every 10 -percentage-point net reduction in serum cholesterol levels (17). In a similar examination of studies concerning the relationship between cholesterol levels and ischemic heart disease, it was found that a $10 \%$ cholesterol reduction would lead to a $25-30 \%$ reduction in mortality from ischemic heart disease over the longterm (18). As both those studies focused on reductions in CHD mortality, it seems likely that the non-fatal incidence of CHD would decrease in a similar fashion if serum cholesterol was reduced across the population.

In an economic evaluation of the health benefits associated with trans-fat-free canola oil, a ratio of a $1 \%$ reduction of cholesterol corresponding to $2-3 \%$ reduction in the incidence of CHD was utilized (9). This result is consistent with the findings of previous research $(17,18)$ and was therefore used as the assumption regarding the reduction in the incidence of $\mathrm{CHD}$ due to decreased blood cholesterol levels resulting from plant sterol consumption in the majority of the sensitivity analysis scenarios.

Step 4: calculation of the reduction in costs associated with CHD due to a reduction in the overall incidence of $C H D$

The final step in the procedure is to quantify the economic benefit resulting from introducing plant sterol-enriched food to the Canadian market. Health Canada has estimated the cost of CHD in Canada 
(7); even though the data are more than a decade old, they are still the most recent and comprehensive available. Since the 1998 data are in nominal dollar amounts but health care costs are subject to inflation, the 1998 amounts require adjustment to more current monetary terms. This adjustment is made using Statistics Canada's consumer price index for health and personal care (19). Adjustment of the 1998 estimate of $\$ 18.472$ billion to 2007 levels yields a new approximation of $\$ 21.176$ billion as the assumed economic cost of CHD in Canada.

The economic cost of disease is generally broken down into direct and indirect components; the former are incurred directly by the health care system with the goal of improving and/or preventing a patient's health status from deteriorating. In the case of heart disease, direct costs include hospital care, drug costs, physician expenses, and other miscellaneous items. Indirect costs are those incurred due to the loss of production arising from disease and include loss of production due to mortality and morbidity costs arising from long- and short-term disability. Table 2 provides a summary of direct and indirect costs attributable to CHD in Canada in both 1998 and inflation-adjusted 2007 dollars. While it seems obvious that a reduction in the level of CHD will lower related costs, is the extent to which these costs will be lowered is less clear. Previous work utilized a directly proportional relationship and assumed that a $1 \%$ reduction in CHD would result in a $1 \%$ reduction in CHD-related costs (9). This type of assumption may oversimplify the health care process and could thus result in an overstatement of the potential cost reduction.

Rather than assuming a proportional cost reduction, it is appropriate to more closely examine the nature of specific cost categories. For example, hospitalization expenditures related to CHD include a sizeable fixed

Table 2. Summary of costs attributed to coronary heart disease in Canada (\$ millions)

\begin{tabular}{lrr}
\hline Cost category & 1998 & 2007 \\
\hline Direct costs & & \\
Hospital & 4161.8 & 4771.0 \\
Drugs & 1772.8 & 2032.3 \\
Physician care & 822.3 & 942.7 \\
Other Direct & 61.2 & 70.2 \\
Total direct costs & 6818.1 & 7816.0 \\
Indirect costs & & \\
Mortality & 8250.0 & 9457.5 \\
Long-term disability & 3151.5 & 3612.8 \\
Short-term Disability & 253.3 & 290.4 \\
Total indirect costs & 11654.8 & 13360.7 \\
Total CHD costs & 18472.9 & 21176.7 \\
\hline
\end{tabular}

Source: Health Canada (7). cost component - the costs of operating a hospital will be incurred regardless of the number of heart attack patients treated at the hospital. Research conducted with respect to the fixed cost variable cost breakdown in hospitals found that hospital costs are approximately $84 \%$ fixed and $16 \%$ variable (20). Accordingly, it is assumed that a reduction in CHD will not result in a reduction in fixed costs of hospitalization, but will cause a proportional reduction in variable hospitalization costs.

Approximating the reduction in drug costs due to CHD assumes that fewer individuals with $\mathrm{CHD}$ will require less medication to treat CHD. Since $49.6 \%$ of drug costs resulting from CHD are for the treatment of hypertension (7), a condition not normally improved as a result of cholesterol reduction, these costs are not reduced in the calculations. The remaining $50.4 \%$ of drug costs associated with CHD are reduced proportionally to the reduction in the overall rate of CHD. Physician care costs associated with CHD are based on physician billings which in turn are derived from patient visits to doctors' offices. It is assumed that if fewer people are afflicted with CHD, fewer visits to physicians for CHD treatment will occur, resulting in less numerous billings and lower health care costs. As such, a 1\% reduction in CHD is assumed to lead to a $1 \%$ reduction in CHDrelated physician costs.

A large component of the miscellaneous CHD-related expenditures has been categorized by Health Canada (7) as services provided by 'other health providers.' For this analysis, the cost of chiropractors $(0.8 \%)$, dental $(26.2 \%)$, and vision $(9.6 \%)$ health professionals were excluded, as such costs are unlikely to be reduced due to a reduction in heart disease. However, the cost of physiotherapists (1\%) and 'all other health professionals' (4.7\%) should be reduced in proportion to the overall CHD reduction. Also ignored in this analysis are costs which are largely fixed and therefore unlikely to decrease with the rate of CHD: expenditures on health research (4.4\%), administration $(6.5 \%)$, and public health $(20.2 \%)$. By contrast, costs that will likely be reduced by a reduction in CHD include ambulance $(4.2 \%)$, home care $(6.2 \%)$, and 'all other health expenditures' $(7.2 \%)$. The final result is that a $1 \%$ decrease in the incidence of CHD is expected to result in a $0.23 \%$ reduction in miscellaneous CHD-related costs.

Mortality, short-term disability, and long-term disability costs were taken to have a directly proportional CHD reduction to reduction in cost relationship. Since the incidence of CHD will decline, it was deemed reasonable to assume the number of people who will die or become disabled as a result of CHD will decline proportionately. As a consequence, the loss of human capital that would ordinarily be incurred as a result of CHD-related death and disability, would not take place; this reduction results in an economic saving. Table 3 summarizes the proportion 
Table 3. Summary of coronary heart disease cost reduction corresponding to a $1 \%$ decrease in incidence of coronary heart disease

\begin{tabular}{lc}
\hline Cost category & Reduction in cost (\%) \\
\hline Hospital & 0.16 \\
Drugs & 0.50 \\
Physician care & 1.00 \\
Other direct & 0.23 \\
Mortality & 1.00 \\
Long-term disability & 1.00 \\
Short-term disability & 1.00 \\
\hline
\end{tabular}

of cost reductions that could be achieved by a reduction in the incidence of CHD.

A number of assumptions are required to approximate the potential reduction in the cost of CHD from introducing phytosterol-fortified foods to the Canadian market. Though these assumptions are based on a review of the peer-reviewed scientific literature, it could be misleading to suggest a single number as best representing the exact economic cost savings. As such, results are reported as a sensitivity analysis; this approach allows the effects of varying model assumptions within the simulation to be gauged. To make the numbers as robust as possible, four scenarios were examined; a description of the sensitivity analysis scenarios is provided below with a summary of the assumptions shown in Table 4.

\section{Results and discussion}

The 'ideal' scenario is one in which the most positive assumptions are used to calculate the greatest possible economic benefit of allowing consumption of plant sterol-enriched food in Canada. It is unlikely that this scenario is achievable in the short-term; however, over the long-term this estimate provides an index of the full potential of the economic savings resulting from the consumption of plant sterol-enriched foods in Canada. The 'optimistic' and 'pessimistic' scenarios make more reasonable assumptions and represent a medium-toshort-term estimate of the potential economic savings possible through plant sterol consumption. The 'very pessimistic' scenario is included to determine the impact on the cost estimations under a worst-case scenario. This scenario could be considered appropriate if the acceptance of phytosterol fortified foods is very low and the efficacy of plant sterols in reducing cholesterol and the health benefits of cholesterol reduction are considerably below expectations.

Table 5 shows the range of values calculated to be representative of the economic savings to Canada from allowing plant sterols in the diet of Canadians. Each of the direct and indirect cost components is represented for the ideal, optimistic, pessimistic, and very pessimistic scenarios described above. Under even the most pessimistic set of assumptions, substantial cost savings would be realized to Canada's publicly funded single-payer health system. Under more reasonable assumptions, the savings become increasingly significant. Although it is unlikely the ideal scenario would be achieved in the short term, it is evident that under a very optimistic set of assumptions, the potential savings become substantial.

The dynamic process from the point in time when plant sterol-enriched foods are introduced to the market to the point in time at which the savings predicted in the various scenarios will occur is difficult to model with certainty. The scenarios presented in this paper represent a static picture at some point in the future. How quickly the product is adopted will determine the speed with which that point is reached. The ideal scenario with a high success rate is more likely to occur at a point in time that is further into the future than the optimistic or pessimistic scenarios which have lower success rates built into their models. While a specific timeline cannot be predicted with certainty, the optimistic scenario requires that Canadians adopt phytosterol-fortified foods in a manner similar to that of Finland, which does not seem unreasonable. Based on the introduction pattern of plant sterol-enriched foods in Finland over the 1990s of 17\%, it would be reasonable to conclude that the impact of introducing plant sterol fortified foods into the Canadian market could be realized in a period of 5-10 years.

\section{General discussion and conclusions}

The results of the simulation indicate that substantial economic savings can result from introducing plant

Table 4. Summary of sensitivity analysis scenario assumptions

\begin{tabular}{lrrrr}
\hline & \multicolumn{3}{c}{ Scenario } \\
\cline { 2 - 5 } Assumption & Ideal (\%) & Optimistic (\%) & Pessimistic (\%) & Very pessimistic (\%) \\
\hline Success rate & 47.0 & 17.0 & 10.0 & 5.0 \\
Cholesterol reduction due to plant sterol consumption & 10.8 & 9.7 & 8.5 & 5.0 \\
CHD reduction due to I\% reduction in serum LDL-cholesterol & 3.0 & 2.5 & 2.0 & 1.0 \\
CHD reduction due to plant sterol consumption & 32.4 & 24.3 & 17.0 & 5.0 \\
\hline
\end{tabular}


Table 5. Reduction in coronary heart disease cost due to consumption of plant sterol enriched foods (\$ millions)

\begin{tabular}{|c|c|c|c|c|}
\hline \multirow[b]{2}{*}{ Cost category } & \multicolumn{4}{|c|}{ Scenario } \\
\hline & Ideal & Optimistic & Pessimistic & Very pessimistic \\
\hline \multicolumn{5}{|l|}{ Direct costs } \\
\hline Hospital & 116.2 & 31.5 & 12.9 & 1.9 \\
\hline Drugs & 155.9 & 42.2 & 17.4 & 2.6 \\
\hline Physician care & 143.5 & 5.9 & 0.1 & $\mathrm{Nil}^{\mathrm{a}}$ \\
\hline Other direct & 2.4 & $\mathrm{Nil}^{\mathrm{a}}$ & $\mathrm{Nil}^{\mathrm{a}}$ & $\mathrm{Nil}^{\mathrm{a}}$ \\
\hline Total direct costs & 418.0 & 79.6 & 30.4 & 4.5 \\
\hline \multicolumn{5}{|l|}{ Indirect costs } \\
\hline Mortality & $\mathrm{I}, 440.2$ & 389.9 & 160.8 & 23.6 \\
\hline Long-term disability & 550.2 & 148.9 & 61.4 & 9.0 \\
\hline Short-term disability & 44.2 & 12.0 & 4.9 & 0.7 \\
\hline Total indirect costs & $2,034.6$ & 550.8 & 227.1 & 33.3 \\
\hline Total CHD costs & $2,452.6$ & 630.4 & 257.5 & 37.8 \\
\hline
\end{tabular}

${ }^{\text {a}}$ Denotes non-zero amounts too small for inclusion.

sterol-enriched foods into the Canadian market. The rising costs of health care exist as a growing concern globally; especially in countries such as Canada where direct costs of treating disease are borne largely by the public health care systems. It has also been found that the cholesterol-lowering effects of plant sterols are additive to other cholesterol lowering strategies such as diet (21) and statin treatment (22). As such, phytosterol enhanced foods can work in concert with lifestyle changes and medication to lower the economic cost of CHD. A possible direction for future research would be to explore the cost effectiveness of reducing CHD through plant sterols in food products compared to statins, which currently enjoy a cost advantage over sterols.

When considering the cost savings presented in this analysis it should be noted that the cost premiums associated with plant sterol-enriched foods have not been accounted for in this valuation. The costs of phytosterol fortified foods will fall outside of the medical system in Canada and thus represent a benefit to the publicly funded health care system. An excessive price premium would result in a lower economic benefit of plant sterol-enriched foods. As the market for phytosterol fortified foods develops and more is known about possible price premiums, this issue should be revisited with a cost-benefit or cost-effectiveness study.

$\mathrm{CHD}$ is a substantial component of the economic burden of illness in Canada and high serum cholesterol is a major risk factor in developing CHD. Functional foods such as those which contain plant sterols have the potential to 'demedicalize' and reduce significant portion of the economic costs associated with CHD in Canada, which in turn would allow health care funds to be allocated more efficiently.

\section{Acknowledgements}

The authors gratefully acknowledge the comments of the Journal editor and two anonymous reviewers.

\section{Conflicts of interest and funding}

The authors have no conflicts of interest. This research was supported by the Coalition for Advancement of Plant Sterols in Canada (CAPSIC).

\section{References}

1. Best M, Duncan C, Van Loon E, Wathen J. Lowering of serum cholesterol by the administration of a plant sterol. Circulation 1954; 10: 201-6.

2. Katan M, Grundy S, Jones P, Law M, Miettnen T, Paoletti R. Efficacy and safety of plant stanols and sterols in the management of blood cholesterol levels. Mayo Clin Proc 2003; 78: 965-78.

3. Ntanios F. Spreads enriched with plant sterol esters lower blood total and LDL cholesterol but not HDL cholesterol levels: a meta-analysis of Unilever studies. Vlaardingen: Unilever Research; 2001.

4. Chen J, Wesley R, Shamburek R, Pucino F, Csako G. Metaanalysis of natural therapies of hyperlipdemia: plant sterols and stanols versus policosanol. Pharmacotherapy 2005; 25: 172-83.

5. AbuMweis S, Barake R, Jones P. Plant sterol/stanols as cholesterol lowering agents: a meta analysis of randomized controlled trials. Food Nutr Res 2008; 54. Available from: http:// www.foodandnutritionresearch.net/index.php/fnr/article/view/ 1811 [cited 1 July 2010].

6. European Food Safety Authority. Plant sterols and blood cholesterol. EFSA J 2008; 781: 1-12. 
7. Health Canada. Economic burden of illness in Canada. Ottawa: Policy Research Division Strategic Policy Directorate Population and Public Health Branch, Health Canada; 1998.

8. Gerber A, Evers T, Haverkamp H, Lauterbach K. Cost-benefit analysis of a plant sterol containing low-fat margarine for cholesterol reduction. Eur J Health Econ 2006; 7: 247-54.

9. Malla S, Hobbs J, Perger O. Valuing the health benefits of a novel functional food. Cdn J Agr Econ 2007; 55: 115-36.

10. French M, Mauskopf J, Teague J, Roland J. Estimating the dollar value of health outcomes from drug-abuse interventions. Medical Care 1996; 34: 890-910.

11. Johnson J, Bootman L. Drug-related morbidity and mortality: a cost-of-illness model. J Mgd Care Pharm 1996; 2: 39-47.

12. Malla S, Hobbs J, Perger O. Estimating the value of health care savings from trans fat-free canola in canada. Food Econ - Acta Ag Scandi, Sec C 2005; 2: 175-84.

13. Statistics Canada. Food statistics. Ottawa: Statistics Canada, Agriculture Division; 2007.

14. Herath D, Cranfield J, Henson S. Who consumes functional foods and nutraceuticals in Canada? Results of cluster analysis of the survey of Canadians' demand for food products supporting health and wellness. Appetite 2008 2006; 51: 256-65.

15. Peng Y, West G, Wang C. Consumer attitudes and acceptance of CLA-enriched dairy products. Cdn J Agr Econ 2006; 54: 663-84.

16. Niva M. Can we predict who adopts health-promoting foods? Users of functional foods in Finland. Scand J Food Nutr 2006; 50: 13-24. Available from: http://www.foodandnutrition research.net/index.php/fnr/article/view/1548 [cited 16 December 2008].
17. Gould A, Rossouw J, Santanello N, Heyse J, Furberg C. Cholesterol reduction yields clinical benefit. Circulation 1995; 91: 2274-82.

18. Law M, Wald N, Thompson S. By how much and how quickly does reduction in serum cholesterol concentration lower risk of ischaemic heart disease? Brit Med J 1994; 308: 367-73.

19. Statistics Canada. Consumer price index: health and personal care. Cansim II Table 126-0021. Ottawa: Statistics Canada; 2007.

20. Roberts R, Frutos P, Ciavarella G, Gussow L, Mensah E, Kampe L, et al. Distribution of variable vs fixed costs of hospital care. JAMA 1999; 281: 644-9.

21. Maki K, Davidson M, Umporowicz D. Lipid responses to plant sterol enriched reduced-fat spreads incorporated into a national cholesterol education program step I diet. Am J Clin Nutr 2001; 74: 33-43.

22. Neil H, Meijer G, Roe L. Randomised controlled trial of use by hypercholesterolaemic patients of a vegetable oil sterol-enriched fat spread. Atherosclerosis 2001; 156: 329-37.

*Peter J.H. Jones

Richardson Centre for Functional Foods and Nutraceuticals

University of Manitoba

196 Innovation Drive, Winnipeg

MB R3T C65, Canada

Tel: +2044748883

Email: peter_jones@umanitoba.ca 